\title{
Non-Poissonian Statistics in a Low-Density Fluid
}

\author{
Paolo Visco, ${ }^{*, \dagger}$ Frédéric van Wijland,, and Emmanuel Trizac ${ }^{\dagger}$ \\ Université Paris-Sud, LPTMS, UMR 8626, Orsay Cedex, F-91405 and CNRS, Orsay, F-91405, and \\ Laboratoire Matière et Systèmes Complexes (CNRS UMR 7057), Université Denis Diderot (Paris VII), \\ 10 rue Alice Domon et Léonie Duquet, 75205 Paris cedex 13, France
}

Received: June 18, 2007

\begin{abstract}
Our interest lies in the collisional statistics in an arbitrary interacting fluid. We show that even in the lowdensity limit and contrary to naive expectation, the number of collisions experienced by a tagged particle in a given time does not obey Poisson law, and that, conversely, the free flight time distribution is not a simple exponential. As an illustration, the hard sphere fluid case is worked out in detail. For this model, we analytically quantify those deviations and successfully compare our predictions against molecular dynamics simulations.
\end{abstract}

The purpose of the present paper is to address, for an arbitrary low-density fluid in a stationary state, basic properties that have been under-appreciated or overlooked, which bear upon the collisional statistics: What is the probability distribution $P(\mathscr{N}$, $t$ ) of the number of collisions $\mathscr{N}$ suffered in equilibrium by a tagged particle over a given duration $t$ ? Conversely, what is the probability distribution function of the free flight time, $P_{\mathrm{FFT}}(\tau)$, again for a tagged particle? Such fundamental questions, relevant in their own right, also have consequences on the evaluation of transport coefficients and, when transposed to the related realm of granular gases, ${ }^{1,2}$ directly quantify dissipation. Our message is that intuitive expectation fails-except in highly untypical cases - as far as the collisional statistics is concerned, for an interacting fluid in and also, arguably less surprisingly, out of equilibrium. All results reported are new, together with the kinetic theory techniques used.

Whereas in a dense fluid, velocity correlations and hydrodynamic effects are responsible for nontrivial collisional statistics, one could naively expect that in the dilute limit where collisions become uncorrelated and molecular chaos is enforced, ${ }^{3,4}$ collisional events define a Poisson process $\mathscr{Q}(\omega)$ so that $P(\mathscr{N}, t)=\exp (-\omega t)(\omega t)^{\mathscr{Y}} \mathfrak{N}$ !, where $\omega$ is the mean collision frequency (i.e., $\langle\mathcal{N}\rangle / t \rightarrow \omega$ at long times, where the brackets denote an ensemble average). The corresponding free flight time distribution would then be

$$
P_{\mathrm{FFT}}(\tau)=-\frac{\mathrm{d}}{\mathrm{d} \tau} P(\mathcal{N}=0, \tau)=\omega \mathrm{e}^{-\omega \tau}
$$

However, as we shall see below (and this seems to have been ignored in the literature ${ }^{5}$ ) such a point of view is flawed. In essence, the collision frequency for a particle with velocity $v$ depends on $\mathbf{v}$ (it generically increases with $v=|\mathbf{v}|$ ), which in turn induces correlations between successive collision times. After general considerations that encompass equilibrium and nonequilibrium steady states, we will show that the collisional statistics is generically non-Poissonian. This is what prompted us to focus on the simplest (analytically tractable) interactions and consider the equilibrium hard sphere fluid for illustrative

* Corresponding author

$\dagger$ Université Paris-Sud.

† Université Denis Diderot. purposes. Such a model is one of the most useful paradigms in statistical mechanics and has played an essential role in the development of the theory of liquids. ${ }^{6}$ It is one of the simplest systems exhibiting a phase transition. Remarkably, it does not only provide a valuable theoretical starting point, but also enjoys direct experimental realizations. ${ }^{7}$ It is therefore surprising that such a well-studied system yields nontrivial properties in a limit where little would have been expected. We will see that, at late times, when $\omega t \gg \mathscr{N}$, the effect of such correlations is that $P(\mathscr{N}$, $t$ ) is of Poissonian form, but with a renormalized frequency $\omega /$ $\sqrt{2}$ instead of $\omega$. This result holds, irrespective of space dimension. In addition, explicit and accurate results will be reported for the number of collision cumulants $\left\langle\hat{N}^{p}\right\rangle_{\mathrm{c}}$. Our analytical predictions will be compared to numerical simulations.

For the sake of simplicity, we begin the analysis by the free flight time distribution $P_{\mathrm{FFT}}(\tau)$. The evolution of a tagged particle in a large stationary homogeneous fluid defines, in the lowdensity limit, a Markov process where the transition rates can be computed from a linearized Boltzmann equation (see, e.g., ref 8). In simplified situations, the velocity-dependent collision rate $r(v)$ can be computed analytically; the inset of Figure 1 shows an illustrative example for hard spheres in equilibrium. Velocities are expressed here in rescaled units, and for equilibrium situations, the velocity distribution function reads, with $d$ the space dimension,

$$
\phi(\mathbf{v})=\frac{1}{\sqrt{2 \pi}^{d}} \mathrm{e}^{-v^{2} / 2}
$$

Out of equilibrium, $\phi$ is a stationary measure. In any case, the mean collision frequency $\omega$ follows from $\phi$ :

$$
\omega=\int \phi(\mathbf{v}) r(v) d \mathbf{v}=\langle r(v)\rangle
$$

From the Markovian property, it follows that the conditional probability of having a free flight time $\tau$ given a velocity $\mathbf{v}$ reads $P_{\mathrm{FFT}}(\tau \mid \mathbf{v})=r(v) \exp (-r(v) \tau)$. To proceed further and obtain $P_{\mathrm{FFT}}(\tau)$ from some average of $P_{\mathrm{FFT}}(\tau \mid \mathbf{v})$, attention must be paid to the fact that the relevant weight to use is not the velocity distribution $\phi(\mathbf{v})$ itself, but the velocity upon collision, $r(v) \phi(\mathbf{v}) / \omega$. The prefactor $r(v)$ biases the distribution toward more energetic events and accounts for the fact that, in a given 


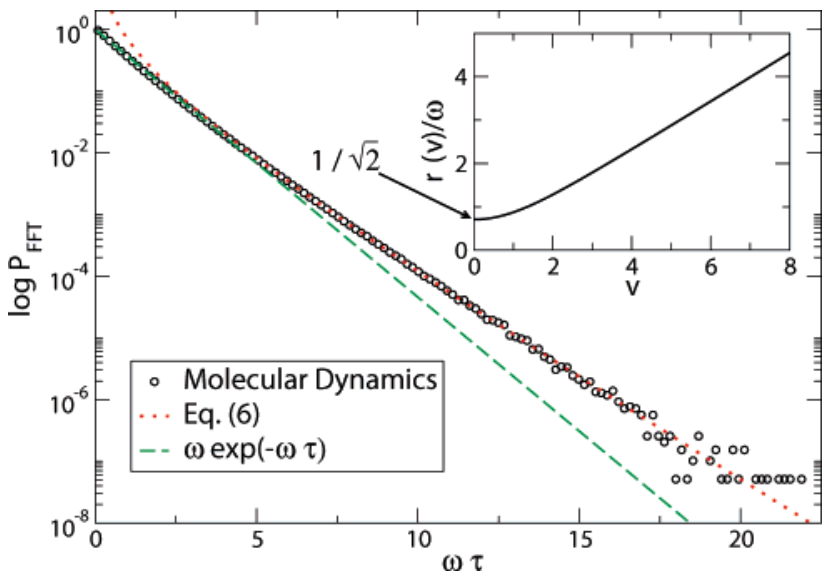

Figure 1. Free flight time distribution as a function of $\omega \tau$, on a linear$\log$ scale, for a two-dimensional hard disc gas $(d=2)$. The circles correspond to the results of molecular dynamics simulations at density $\rho=0.04 \sigma^{-2}$, where $\sigma$ is the disc's diameter, with $N=1000$ particles. The dotted line shows the prediction of eq 6 , while eq 1 is shown with a dashed line. The inset shows the tagged collision frequency $r(v) / \omega$ versus $v$, also for $d=2$. The value $1 / \sqrt{2}$ at $v=0$, indicated with an arrow, is $d$-independent.

time interval, a particle with a larger than typical velocity will experience more collisions. We therefore obtain

$$
P_{\mathrm{FFT}}(\tau)=\int d \mathbf{v} \frac{r^{2}(v)}{\omega} \mathrm{e}^{-r(v) \tau} \phi(\mathbf{v})
$$

This expression explicitly differs from the result reported in ref 9 , where $r^{2} / \omega$ is replaced by $r$ (in other words, the weight used in ref 9 is $\phi$ and not $r \phi / \omega)$. To see why such an approach is incorrect, one can compute the mean collision time $\langle\tau\rangle=$ $\int \tau P_{\mathrm{FFT}}(\tau) d \tau$, which should be equal to $1 / \omega$. This is indeed the case with the distribution given in eq 4 , whereas the formula of ref 9 gives $\langle\tau\rangle=\langle 1 / r\rangle$, which differs from $1 /\langle r\rangle=1 / \omega \cdot{ }^{10}$ More importantly, upon neglecting the $v$ dependence of the rate $r$ (i.e., assuming $r=\omega$ ), the integral in eq 4 is readily integrated and yields expression 1 for $P_{\mathrm{FFT}}$. It has been shown that a $v$-independent collision rate corresponds to particles interacting via an inverse power law pair potential with exponent $2 d-2,{ }^{4}$ which defines the so-called Maxwell model, ${ }^{11}$ a particularly convenient framework in kinetic theory. Maxwell molecules are nevertheless highly untypical, and, for any other fluid, $r$ depends on $v$ so that eq 4 cannot be exponential. We therefore conclude here that the collisional statistics is, in general, non-Poissonian, except for Maxwell molecules where successive collisions turn out to be uncorrelated. We will clarify below the conditions for the occurrence of correlations, and show that, while the $v$ dependence of $r$ is a necessary condition for non-Poissonian behavior, it is, in general, not sufficient.

After the previous qualitative remarks, our goal is to quantify the deviations for Poissonian behavior, and to this end, we hereafter consider the prototypical hard sphere model where the frequency $r(v)$ takes the form ${ }^{8}$

$r(v)=\frac{\omega}{\sqrt{2}}\left(\frac{v^{2}}{d}{ }_{1} F_{1}\left(\frac{1}{2}, 1+\frac{d}{2},-\frac{v^{2}}{2}\right)+\mathrm{e}^{-v^{2} / 2}{ }_{1} F_{1}\left(\frac{d-1}{2}, \frac{d}{2}, \frac{v^{2}}{2}\right)\right)$

where ${ }_{1} F_{1}$ denotes a confluent hypergeometric function of the first kind. Although a closed-form expression cannot be obtained for $P_{\mathrm{FFT}}(\tau)$ because of the lack of simplicity of the collision rate $r(v)$, finding the large $\tau$ behavior calls for a saddle point approximation for the integral appearing in eq 4 , which yields

$$
P_{\mathrm{FFT}}(\tau) \stackrel{\omega \tau \gg 1}{\sim} \exp \left(-\frac{\omega \tau}{\sqrt{2}}\right) \frac{\omega}{2}\left(1-\frac{2}{d}+\frac{\omega \tau}{\sqrt{2} d}\right)^{-d / 2}
$$

Interestingly, to leading order, $\tau$ is distributed exponentially, as naively expected [see eq 1], but with a renormalized rate $\omega /$ $\sqrt{2}$. The validity of expression 6 is illustrated in Figure 1, which displays the results of numerical simulations. We have checked that the molecular dynamics data in Figure 1 precisely coincide with the numerical integration of eq 4 for all velocities (not shown).

We also note here that very similar considerations hold for the distribution of path lengths: for a particle with velocity $\mathbf{v}$, the free flight distance (FFD) $l$ travelled in a time $t$ is $l=v t$, so that $P_{\mathrm{FFD}}(l \mid \mathbf{v})=v^{-1} P_{\mathrm{FFT}}\left(l v^{-1} \mid \mathbf{v}\right) . P_{\mathrm{FFD}}$ then follows from the counterpart of eq 4

$$
P_{\mathrm{FFD}}(l)=\int d \mathbf{v} \frac{r^{2}(v)}{\omega v} \exp \left(-\frac{r(v)}{v} l\right) \phi(\mathbf{v})
$$

The resulting probability density is not a simple exponential, at variance with a claim sometimes found in the literature (see, e.g., refs 12 and 13). However, a saddle point computation akin to that leading to eq 6 provides here the long distance behavior of $P_{\mathrm{FFD}}$, which is governed by the minimum of the function $r(v) / v{ }^{14}$ This leads (a) to $P_{\mathrm{FFD}}(l) \propto \exp \left(-\omega\langle v\rangle^{-1} l / \sqrt{2}\right)$ for $l$ much larger than the mean free path, and (b) to the remark that, for the Maxwell model with a $v$-independent rate $r$, the minimum of $r(v) / v$ is reached for $v \rightarrow \infty$ and vanishes, which leads to a non-exponential large $l$ limiting behavior. ${ }^{15}$

We now turn to the related but more complex question of the number of collisions. We introduce the joint probability $f(\mathbf{v}$, $\mathcal{N}, t$ ) of having velocity $\mathbf{v}$ and having suffered $\mathcal{N}$ collisions in a time window $t$, for our tagged particle. The corresponding time evolution, again in the dilute limit, follows from the linear Boltzmann-like equation

$$
\begin{aligned}
& \partial_{f} f\left(\mathbf{v}_{1}, \mathcal{N}, t\right)= \\
& \int \mathrm{d} \mathbf{v}_{2} \int \mathrm{d} \hat{\sigma}\left(\mathbf{v}_{12} \cdot \hat{\sigma}\right) \theta\left(\mathbf{v}_{12} \cdot \hat{\sigma}\right)\left[f\left(\mathbf{v}_{1}^{* *}, \mathcal{N}-1, t\right) \phi\left(\mathbf{v}_{2}^{* *}\right)-\right. \\
& \left.f\left(\mathbf{v}_{1}, \mathcal{N}, t\right) \phi\left(\mathbf{v}_{2}\right)\right]
\end{aligned}
$$

where $\theta$ is the Heaviside function, $\mathbf{v}_{12}$ is the relative velocity, $\hat{\sigma}$ is a unit vector, and the $* *$ superscript refers to precollisional velocities: $\mathbf{v}_{1}^{* *}=\mathbf{v}_{1}-\left(\mathbf{v}_{12} \bullet \hat{\sigma}\right) \hat{\sigma}$ and $\mathbf{v}_{2}^{* *}=\mathbf{v}_{2}+\left(\mathbf{v}_{12} \bullet \hat{\sigma}\right) \hat{\sigma}$. The latter equation encodes a full description of the collisional statistics for the tagged particle, in the low-density limit. Here again we stress that such an analytical approach can be extended to other interaction potentials, leading to the already mentioned remark that, apart from Maxwell molecules, it does not admit a Poisson solution. Moreover, the above equation does not provide a stationary solution in the long time limit, because of the time-dependent behavior of the collision number $\mathscr{N}$. More precisely, we expect the large time dependence to be exponential, as a consequence of the linear character of the equation. For analytical progress, it becomes convenient to introduce the generating function $\hat{f}$ through

$$
\hat{f}(\mathbf{v}, \lambda, t)=\sum_{\lambda=0}^{\infty} \mathrm{e}^{-\lambda /} f(\mathbf{v}, \mathcal{N}, t)
$$

Of course, upon summing eq 8 over all possible values of $\mathscr{N}$, in the equilibrium state, one recovers $\sum_{\mathcal{N}=0}^{\infty} f(\mathbf{v}, \mathcal{N})=$ $\hat{f}(\mathbf{v}, 0, t)=\phi(\mathbf{v})$, the Maxwell-Boltzmann distribution (eq 2). 


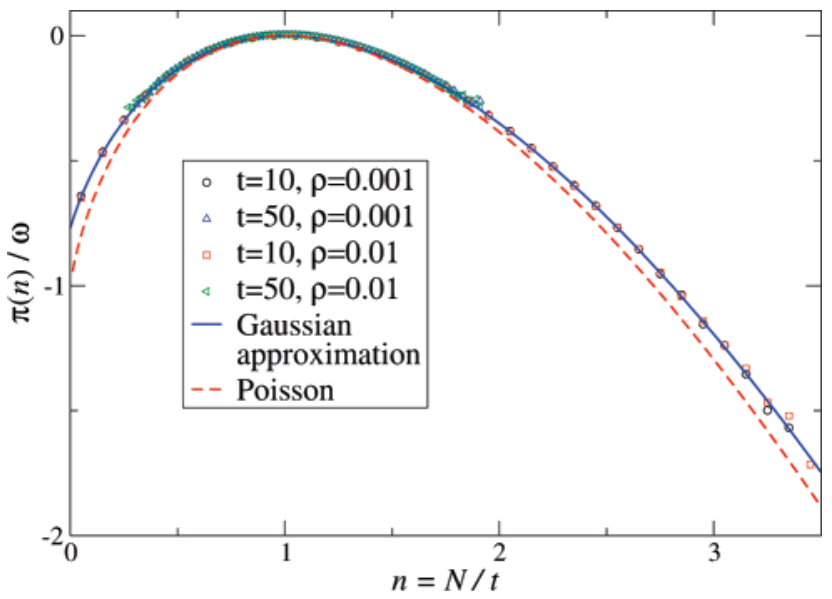

Figure 2. Large deviation function $\pi(n)$ characteristic of the longtime behavior of the probability $P(\mathscr{N}, t)$ of suffering $\mathcal{N}$ collisions in a time $t$ (and defined by $\pi(\mathcal{N} / t) \sim t^{-1} \log P(\mathcal{I}, t)$ for $\omega t \gg 1$ ). The symbols correspond to molecular dynamics measurements for a hard disc system with $N=1000$ particles at two different but low densities. The solid line shows the Gaussian result following from eq 16, while the dashed line is the large deviation function $\pi(n)=n-n \log (n / \omega)$ - $\omega$ associated to the Poisson law $\mathscr{Q}(\omega)$. On the graph, the time scale has been set by the choice $\omega=1$.

TABLE 1: Cumulants for the Number of Collisions $\mathcal{N}$ from Molecular Dynamics Simulations (Performed on a

Two-Dimensional System with Reduced Density $\rho \sigma^{2}=0.04$ ), and Comparison with Both the Poisson $\mathscr{P}(\omega)$ Result and the Gaussian Approximation (17)

\begin{tabular}{lcll}
\hline & $\langle N\rangle_{\mathrm{d}} / t$ & $\left\langle N^{2}\right\rangle_{\mathrm{c}} / t$ & $\left\langle N^{3}\right\rangle_{\mathrm{c}} / t$ \\
\hline$\omega t=10$ & 1. & 1.123 & 1.129 \\
$\omega t=50$ & 1. & 1.135 & 1.105 \\
Poisson & 1 & 1 & 1 \\
Gaussian & 1 & 1.125 & 1.129
\end{tabular}

It then appears that the cumulant generating function $\mu(\lambda)$, such that,in the large time limit,

$$
\left.\left\langle\mathcal{N}^{p}\right\rangle_{\mathrm{c}}^{\omega t \gg 1} \sim t(-1)^{p} \frac{\partial^{p} \mu}{\partial \lambda^{p}}\right|_{\lambda=0}
$$

is the largest eigenvalue of an evolution operator that straightforwardly follows from eq $8 .{ }^{15}$ Furthermore, $\mu(\lambda)$ is directly related to the large time behavior of $P(\mathcal{N}, t)$ through its large deviation function $\pi$, defined as

$$
P(\mathscr{N}, t) \stackrel{\omega t \gg 1}{\sim} \mathrm{e}^{t \pi(n)}
$$

where $n=\mathcal{N} / t$. Indeed, $\pi$ is the Legendre transform of $\mu$ :

$$
\pi(n)=\min _{\lambda}(\mu(\lambda)+\lambda n)
$$

The quantity $\mu(\lambda)$ therefore bears important physical information, and has been the technical focus of our study.

From perturbation theory, we have obtained the behavior of $\mu$ at large $\lambda$ in the form

$$
\mu(\lambda) \sim \frac{\omega}{\sqrt{2}}\left(\mathrm{e}^{-\lambda}-1\right)+\theta\left(\mathrm{e}^{-2 \lambda}\right)
$$

which implies that

$$
P(\mathcal{N}, t) \sim \frac{\mathrm{e}^{-\omega t /} \sqrt{2}}{\mathcal{N} !}\left(\frac{\omega t}{\sqrt{2}}\right)^{\mathcal{N}} \text {, for } \mathcal{N} \ll \omega t
$$

For this Poissonian behavior $\mathscr{Q}(\omega / \sqrt{2})$, the large deviation function easily follows:

$$
\pi(n)=n-n \log (n \sqrt{2} / \omega)-\omega / \sqrt{2}
$$

We note that eq 14 with $\mathscr{N}=0$ is compatible with the time integral of the leading exponential order of free flight time distribution given in eq 6 , as it should.

However, the dependence embodied in eqs 13 and 14 follows from a large $\lambda$ expansion and only holds for $\mathcal{N} \ll \omega t$ (hence, $n / \omega \rightarrow 0)$. It is therefore not indicative of the typical behavior, for which it is more useful to consider the low $\lambda$ limit. We then dwell on the remark made after eq 9 that, for $\lambda=0$, we have $\hat{f}(\mathbf{v}, 0, t)=\phi(\mathbf{v})$, which leads to the approximation scheme where $\hat{f}(\mathbf{v}, \lambda, t)$ is taken as a Gaussian with temperature $T(\lambda)$ and the requirement that $T(0)=1 .{ }^{16}$ This approximation is the lowest order of a more systematic expansion (see ref 15), but it provides useful and reasonably accurate information given its simplicity. The idea is to project the Boltzmann eq 8 governing the evolution of $\hat{f}(\mathbf{v}, \lambda, t)$ onto the first two velocity moments. This leads to

$$
\mu(\lambda)=-\frac{\omega}{\sqrt{2}}\left(1-\mathrm{e}^{-\lambda}\right) \sqrt{1+\frac{T(\lambda)}{T_{0}}}
$$

with

$$
T(\lambda)=\frac{\sqrt{2} T_{0}}{\sqrt{1+\mathrm{e}^{\lambda}}}
$$

It can be checked that $\mu(\infty)=-\omega / \sqrt{2}$, as implied by eq 13 . The corresponding first three cumulants follow from eq 10 and read, irrespective of dimension $d$,

$$
\begin{gathered}
\frac{\langle\mathcal{N}\rangle_{\mathrm{c}}}{\omega t}=1 \\
\frac{\left\langle\mathcal{N}^{2}\right\rangle_{\mathrm{c}}}{\omega t}=\frac{9}{8} \\
\frac{\left\langle\mathcal{N}^{3}\right\rangle_{\mathrm{c}}}{\omega t}=\frac{289}{256}
\end{gathered}
$$

These values are compared to molecular dynamics simulation data in Table 1. Moreover, the large deviation function $\pi(n)$ of collisions, which follows from eq 12, appears to be in excellent agreement with its molecular dynamics counterpart (see Figure 2).

At this point, it proves instructive to consider the Lorentz gas with only one mobile particle and a collection of spherical fixed scatterers. One readily gets $r(v) \propto v$, but the collisional statistics is nevertheless Poissonian. The reason is that the velocity modulus of the mobile particle is constant along the trajectory: in other words, there is no thermalization. It therefore appears that non-Poissonian behavior arises from two key properties that induce collisional correlations: first, the collision rate depends on the velocities, and, second, the particle thermalizes to some stationary nonsingular measure. These criteria also apply out of equilibrium.

In conclusion, we have shown that the statistics of the number of collisions $\mathcal{N}$ experienced by a tagged particle in a low-density homogeneous and stationary fluid, in or out of equilibrium, is a subtler quantity than it might seem. Our general statements have been illustrated with the hard sphere fluid. For the 
distributions of both $\mathscr{N}$ and the related free flight time (for which several incorrect results may be found in the literature), we have quantified the corresponding non-Poissonian behavior that follows from the simple physical ingredient that a particle with a high velocity statistically collides more often than a typical particle. A key quantity in the theoretical analysis is the cumulant generating function $\mu(\lambda)$, which can be computed explicitly for large $\lambda$ and approximately for small $\lambda$. The Gaussian ansatz worked out here can be considered as the lowest order of a systematic expansion. The resulting analytical predictions have been confronted against molecular dynamics numerical simulations, with a very good agreement. These numerical results show that the deviations from Poisson behavior $\mathscr{P}(\omega)$, although not dramatic (which may be the reason why they are under-documented in the literature) are nevertheless clearly observable. In particular, we have obtained the a priori surprising result that, for long times, the distribution of $\mathcal{N}$ is Poissonian, but with a "dressed" rate $\omega / \sqrt{2}$. Conversely, the distribution of free flight time $\tau$ is exponential, with a behavior $\propto \exp (-\omega \tau / \sqrt{2})$.

Acknowledgment. We would like to thank J. Piasecki, J. M. J. van Leeuwen, M. H. Ernst, D. Frenkel and H. van Beijeren for useful discussions. This work was supported by the French Ministry of Education through a JCJC ANR grant.

\section{References and Notes}

(1) Brilliantov, N. V.; Poschel, T. Kinetic Theory of Granular Gases; Oxford University Press: Oxford, 2004.
(2) Barrat, A.; Trizac, E.; Ernst, M. H. J. Phys.: Condens. Matter 2005, 17, S2429.

(3) Résibois, P.; de Leener, M. Classical Kinetic Theory of Fluids; John Wiley and Sons: New York, 1977.

(4) Cercignani, C. Rarefied Gas Dynamics; Cambridge University Press: Cambridge, U.K., 2000.

(5) The fact that $\mathcal{N}$ is not a Poisson variable has already been reported in the literature (see, e.g., Lue, L. J. Chem. Phys. 2005, 122, 044513 for numerical data on hard spheres), but to the best of our knowledge, no analytical results have been obtained.

(6) Hansen, J. P.; MacDonald, I. R. Theory of Simple Liquids, 3rd ed.; Academic Press: New York, 2006.

(7) Pusey, P. N.; van Megen, W.; Bartlett, P.; Ackerson, B. J.; Rarity, J. G.; Underwood, S. M. Phys. Rev. Lett. 1989, 63, 2753. Eldridge, M. D. Madden, P. A.; Frenkel, D. Nature 1993, 365, 35. Hunt, N.; Jardine, R. R.; Bartlett, P. Phys. Rev. E 2000, 62, 900. Yethiraj, A; van Blaaderen, A. Nature 2003, 421, 513

(8) Puglisi, A.; Visco, P.; Trizac, E.; van Wijland, F. Phys. Rev. E 2006, 73, 021301

(9) Wiegel, F. W.; Michels, J. P. J. Chem. Phys. Lett. 1976, 40, 23.

(10) This problem also pertains to the definition of time in directsimulation Monte Carlo algorithms, as discussed in Koura, K. Phys. Fluids 1986, 29, 3509 .

(11) Ernst, M. H. Phys. Rep. 1981, 78, 1.

(12) Hecht, C. E. Statistical Thermodynamics and Kinetic Theory; Dover Publications: New York, 1998

(13) Blair, D. L.; Kudrolli, A. Phys. Rev. E 2003, 67, 041301.

(14) Conversely, the minimum of $r(v)$ is the relevant quantity for the long-time behavior of eq 4; it is reached at $v=0$ (see the inset of Figure 1 ), where $r(0)=\omega / \sqrt{2}$. This explains the $\sqrt{2}$ factors appearing in eq 6 .

(15) Visco, P.; van Wijland, F.; Trizac, E. To be submitted for publication.

(16) More precisely, it can be shown that, at late times, $\hat{f}(\mathbf{v}, \lambda, t)$ takes the asymptotic form $\exp [\mu(\lambda) t] \tilde{f}(\mathbf{v}, \lambda)$, so that the time dependence factorizes from the $\mathbf{v}$ variable, which explains why $T(\lambda)$ is $t$-independent. The requirement that $\mu(0)=0$ ensures that $\hat{f}(\mathbf{v}, 0, t)$ is also time independent. 